\title{
MINIMIZING TIMES BETWEEN BOUNDARY POINTS ON RECTANGULAR POOLS
}

\author{
TONJA MIICK AND TOM RICHMOND ${ }^{1}$
}

\begin{abstract}
The well-known "do dogs know calculus" problem optimizes the travel time from an onshore dog to an offshore stick, given different running and swimming speeds and a straight coastline. Here, we optimize the travel time between two points on the boundary of a rectangular swimming pool, assuming that running speed along the edge differs from the swimming speed.
\end{abstract}

A common calculus problem is to minimize the time from point $A$ to point $B$ if part of the distance can be covered at a faster speed than the rest. If a $\operatorname{dog}$ is at point $A$ on a straight shoreline and a stick is at point $B$ in the ocean, the $\operatorname{dog}$ may wish to minimize the time from $A$ to $B$ if the running speed $r$ is greater than the swimming speed $s$ (see "Do Dogs Know Calculus?" [5]). With a straight boundary between media, the problem is equivalent to Snell's law in optics, based on Fermat's principle that light takes the fastest route from $A$ to $B$ (see $[2,6]$ ).

An interesting variation is to consider getting from $A$ to $B$ if both points lie on the boundary of a circular pond. It is an easy exercise in calculus to show that the optimal path may run around the border from $A$ to $B$ or may swim directly from $A$ to $B$, but will never mix running and swimming.

Here, we consider the problem of getting from $A$ to $B$ as quickly as possible if $A$ and $B$ both lie on the boundary of a rectangular pool. These results are based on [4]. Some of the results of Section 1 have appeared in [3]. A special case where $A$ and $B$ lie outside the boundary of a rectangular pool is considered in [1].

\section{The 2-Sided CASE}

Suppose we are traveling from a point $A=(0, a)$ on an edge of a rectangular pool $[0, \infty) \times[0, \infty)$ to point $B=(b, 0)$ on an adjacent edge of the pool, where $a$ and $b$ are positive. By scaling and reflecting, we may assume $a \geq b=1$ and the swim speed $s$ is 1 unit. Then the running speed $r$ may be thought of as the ratio of the running speed to swimming speed. If the

\footnotetext{
${ }^{1}$ Corresponding author.
} 


\section{MINIMIZING TIMES BETWEEN BOUNDARY POINTS ON POOLS}

running speed $r$ is less than $s=1$, then the all swimming path is shortest and fastest, and thus optimal. So, we will assume $r>s=1$. The most general path from $A$ to $B$ to consider is one as shown in Figure 1 which runs a bit from $A$ along the poolside before swimming across a corner of the pool and then running on to $B$ along the terminal side.

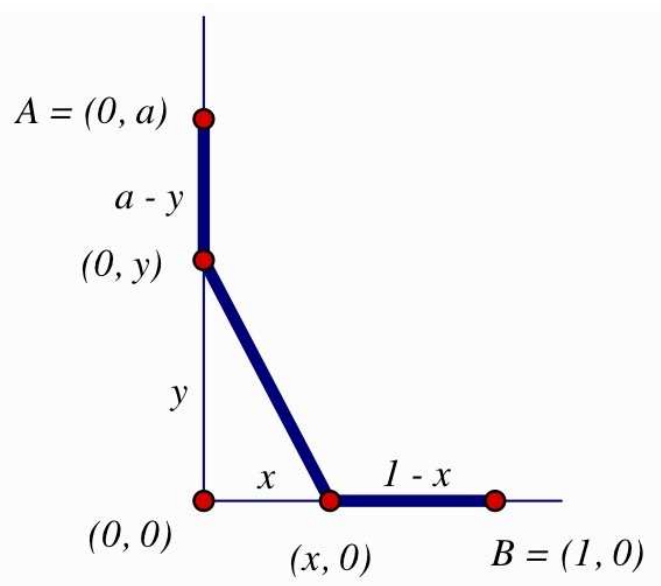

FIGURE 1. 2-sided rectangle path possibilities.

With the points as labeled in Figure 1, the time to traverse this path is

$$
T(x, y)=\frac{1-x+a-y}{r}+\sqrt{x^{2}+y^{2}},
$$

where $(x, y) \in[0,1] \times[0, a]$. This time will be minimized either at a point on the boundary of the domain $[0,1] \times[0, a]$ or at a critical point.

The critical points occur where the partial derivates $T_{x}(x, y)$ and $T_{y}(x, y)$ are simultaneously zero, which occurs when $x=y$. Substituting $x=y$ into $T_{x}(x, y)=0$ leads to $r=\sqrt{2}$. With $r=\sqrt{2}$, the time function $T(x, y)$ for $x=y$ becomes $T(x, x)=\frac{1+a}{\sqrt{2}}$, a constant function, so any such path cutting the corner at $45^{\circ}$ angles will take the same time. Indeed, this holds for $x=y=0$ which corresponds to the all-running path. Thus, the optimal paths occurring at critical points only occur in the special case $r=\sqrt{2}$, when we may run all the way or cut across the corner at $45^{\circ}$ angles.

If $r \neq \sqrt{2}$, the minimal time will correspond to a boundary point on the domain, and even if $r=\sqrt{2}$, we must compare the value at the critical points to the values on the boundary of the domain. We will consider the cases $1<r<\sqrt{2}, r=\sqrt{2}$, and $r>\sqrt{2}$ separately. 


\section{T. MIICK AND T. RICHMOND}

Referring to Figure 1, it is easy to see that no minimum can occur for boundary points of form $(0, y)$ for $y \in(0, a]$ or of form $(x, 0)$ for $x \in(0,1]$, for such points correspond to paths which follow the edge of the pool all the way from $A$ to $B$ but traverse part of an edge swimming, which is slower than running. This does not exclude the possibility of a minimum at $(x, y)=(0,0)$, which corresponds to the all running path.

For points $(1, y)$ for $y \in[0, a]$ on the right boundary, we have $T(x, y)=$ $T(1, y)=T_{r}(y)=\frac{a-y}{r}+\sqrt{1+y^{2}}$, and $T_{r}^{\prime}(y)>0$ if and only if $y>\frac{1}{\sqrt{r^{2}-1}}$. Thus, $T_{r}(y)$ decreases until $y=\frac{1}{\sqrt{r^{2}-1}}$ and increases after that, so there will be a minimum along this edge at this critical point if it falls in the domain $y \in[0, a]$, and otherwise the minimum along this edge will occur when $y=a$, the all-swimming path. So, if the minimum of $T(x, y)$ occurs on this edge, it is either $T\left(1, \frac{1}{\sqrt{r^{2}-1}}\right)$ corresponding to a run-swim path or $T(1, a)$ corresponding to the swim path.

We note that the critical point $\frac{1}{\sqrt{r^{2}-1}}$ falls in the domain $[0, a]$ if and only if $a \geq \frac{1}{\sqrt{r^{2}-1}}$ if and only if $r \geq \sqrt{1+\frac{1}{a}}$. Since $a \geq 1$, this critical point always occurs in the domain if $r \geq \sqrt{2}$.

The case for top boundary points of form $(x, a)$ for $x \in[0,1]$ is dual, with $T(x, y)=T(x, a)=T_{t}(x)=\frac{1-x}{r}+\sqrt{x^{2}+a^{2}}$ decreasing along the edge $[0,1] \times\{a\}$ until $x=\frac{a}{\sqrt{r^{2}-1}}$, giving a local minimum along this edge at this critical point if it is in the domain $x \in[0,1]$, and at $x=1$ otherwise, giving the all-swimming path. So, if the minimum of $T(x, y)$ occurs on this edge, it is either $T\left(\frac{a}{\sqrt{r^{2}-1}}, a\right)$ corresponding to a swim-run path, or $T(1, a)$ arising from the swimming path.

We note that the critical point $\frac{a}{\sqrt{r^{2}-1}}$ falls in the domain $[0,1]$ if and only if $a \leq \sqrt{r^{2}-1}$ if and only if $r \geq \sqrt{a+1}$. Since $a \geq 1$, this critical point will not fall in the domain if $r<\sqrt{2}$.

Thus, there are four possibilities for the minimum for $T(x, y)$ along the boundary of the domain $[0,1] \times[0, a]$, which we will view and label as functions of $a$. The paths giving rise to these minimal times, their associated time functions, and their domains are given in Figure 2.

The following lemma will help us determine which of these, or the paths occurring at the critical points for $r=\sqrt{2}$, actually gives the minimum time.

Lemma 1.1. For $a \in[1, \infty)$,

(a) $R(a) \leq R S(a)$ if and only if $r \geq \sqrt{2}$, with strict inequality if $r>$ $\sqrt{2}$.

(b) $S R(a) \leq S(a)$ and equality holds only at $a=\sqrt{r^{2}-1}$.

(c) $R S(a) \leq S(a)$ and equality holds only at $a=\frac{1}{\sqrt{r^{2}-1}}$. 


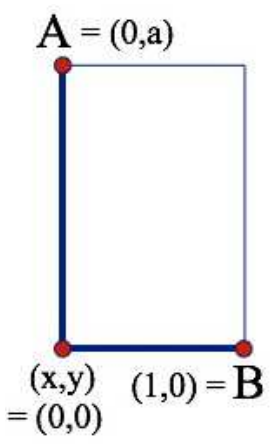

the Run path $R(a)$ $=\frac{1+a}{r}$ for $a \geq 1$

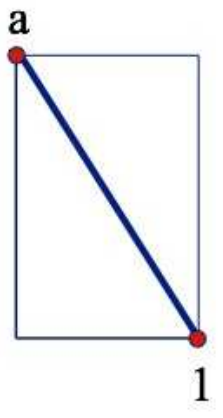

the Swim path $S(a)$ $=\sqrt{a^{2}+1}$ for $a \geq 1$

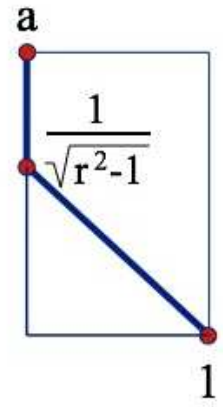

the Run-Swim path $R S(a)$ $=\frac{1}{r}\left(a+\sqrt{r^{2}-1}\right)$ for $a \geq \frac{1}{\sqrt{r^{2}-1}}$

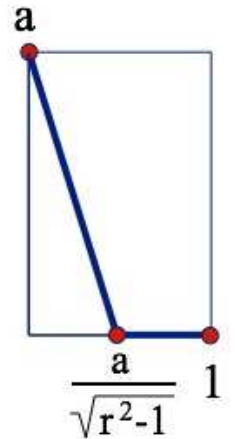

the Swim-Run path $S R(a)$ $=\frac{1}{r}\left(1+a \sqrt{r^{2}-1}\right)$ for $a \in\left[1, \sqrt{r^{2}-1}\right]$

Figure 2. Possible optimal paths on the boundary of the domain and their time functions.

(d) For $a>1, S R(a)<R S(a)$ if and only if $r<\sqrt{2}$.

(e) $S(a)<R(a)$ if and only if $r<\sqrt{2}$ and $\frac{1-r \sqrt{2-r^{2}}}{r^{2}-1}<a<\frac{1+r \sqrt{2-r^{2}}}{r^{2}-1}$.

Proof. (a) As functions of $a, R$ and $R S$ are parallel lines with slope $\frac{1}{r}$, and $R \leq R S$ if and only if $R(0) \leq R S(0)$, that is, if and only if $r \geq \sqrt{2}$, with strict inequality if $r>\sqrt{2}$.

(b)-(c) It is easy to verify that the line $S R(a)$ is tangent to the hyperbola $S(a)$ at $a=\sqrt{r^{2}-1}$, and $R S(a)$ is tangent to $S(a)$ at $a=\frac{1}{\sqrt{r^{2}-1}}$. In particular, since the hyperbola $S(a)$ is concave up, these tangent lines lie below $S(a)$, and thus the all swim solution $S$ will never be minimal, except possibly at the point of tangency, if either of the critical points giving rise to the lines $R S$ and $S R$ actually falls in the domain of $T(x, y)$.

(d) The lines $R S$ and $S R$ have slopes $\frac{1}{r}$ and $\frac{\sqrt{r^{2}-1}}{r}$, respectively, and intersect at $a=1$. Thus, $R S$ has the larger slope if and only if $1>\sqrt{r^{2}-1}$, or equivalently if and only if $r<\sqrt{2}$. Thus, on the domain $a>1$, we have $S R<R S$ if and only if $r<\sqrt{2}$.

(e) The inequality $S(a)<R(a)$ leads to $p(a)=a^{2}\left(1-r^{2}\right)+2 a+1-r^{2}>0$. Now $p(a)=0$ at $\frac{1 \pm r \sqrt{2-r^{2}}}{r^{2}-1}$ if the discriminant is nonnegative, that is, if $r<\sqrt{2}$. Since $r>1, p(a)$ is a parabola opening downward, and is either never positive or only positive between the zeros if $r<\sqrt{2}$. 


\section{T. MIICK AND T. RICHMOND}

Theorem 1.2. Suppose $A=(0, a)$ and $B=(b, 0)$ with $a \geq b=1$ are on adjacent edges of a rectangular pool $[0, \infty) \times[0, \infty)$, where the running speed is $r>1$ and the swimming speed is 1 .

(a) For $1<r<\sqrt{2}$, the run-swim path from $A$ to $\left(0, \frac{1}{\sqrt{r^{2}-1}}\right)$ to $B$ is optimal if $\frac{1}{\sqrt{r^{2}-1}} \leq a$, and the swim path form $A$ to $B$ is optimal if $\frac{1}{\sqrt{r^{2}-1}}>a$.

(b) For $r=\sqrt{2}$, the run path from $A$ to $(0,0)$ to $B$ is optimal, as are any paths which swim across a $45^{\circ}$ corner.

(c) For $r>\sqrt{2}$, the run path from $A$ to $(0,0)$ to $B$ is optimal.

Proof. For (a), suppose $1<r<\sqrt{2}$. Then $S R(a)=T\left(\frac{a}{\sqrt{r^{2}-1}}, a\right)$ is not valid since the critical point $x=\frac{a}{\sqrt{r^{2}-1}}$ falls outside the domain $x \in[0,1]$. We have $R S(a)<R(a)$ by Lemma 1.1(a), and $R S(a) \leq S(a)$ by Lemma 1.1(c), so, if $R S(a)$ is a valid option, then it gives the minimum. We will show that the interval $a \in\left[1, \frac{1}{\sqrt{r^{2}-1}}\right)=\left[1, \frac{\sqrt{r^{2}-1}}{r^{2}-1}\right)$ where $R S(a)$ is not a valid option falls entirely in the interval $\left(\frac{1-r \sqrt{2-r^{2}}}{r^{2}-1}, \frac{1+r \sqrt{2-r^{2}}}{r^{2}-1}\right)$ where, by Lemma 1.1(e), $S(a)<R(a)$. For $1<r<\sqrt{2}$, we have $\sqrt{r^{2}-1}<1<1+r \sqrt{2-r^{2}}$ so $\frac{\sqrt{r^{2}-1}}{r^{2}-1}<\frac{1+r \sqrt{2-r^{2}}}{r^{2}-1}$. Also, $\frac{1-r \sqrt{2-r^{2}}}{r^{2}-1}<1$ is equivalent to $f(r)=\frac{2}{r}<\sqrt{2-r^{2}}+r=g(r)$, which holds for $r \in(1, \sqrt{2})$ since $f$ and $g$ agree at the endpoints of this interval and $f$ is concave up, and $g$ is concave down on the interval. Thus, $R S(a)=T\left(1, \frac{1}{\sqrt{r^{2}-1}}\right)$ is the minimum where it is valid, and $S(a)$ is minimum otherwise.

For (b), suppose $r=\sqrt{2}$. Then $R S(a)=S R(a)=R(a) \leq S(a)$, Thus, the minimal time corresponding to points $(x, y)$ on the boundary of the domain $[0,1] \times[0, a]$ of $T(x, y)$ is produced by the all running path $(R(a)=$ $T(0,0))$. But recall that this case $r=\sqrt{2}$ was the only case with interior critical points. They corresponded to run-swim-run paths cutting any $45^{\circ}$ corner off the pool. Each of these requires the same time, so each will give the minimum. The minima corresponding to $R S(a)=T\left(1, \frac{1}{\sqrt{r^{2}-1}}\right)$ and $S R(1)$ involve swimming across a $45^{\circ}$ corner, and thus are accounted for.

For (c), suppose $r>\sqrt{2}$. Then by Lemma 1.1, we have $R(a)<R S(a) \leq$ $S R(a) \leq S(a)$, so the all running path $R(a)$ is minimum.

The discussion above assumed $a \geq b=1$. If we drop the restriction that $b=1$, then scaling by $b$ simply has the effect of moving the potential critical point $y=\frac{1}{\sqrt{r^{2}-1}}$ on the long edge corresponding to $R S(a)$ to $y=\frac{b}{\sqrt{r^{2}-1}}$. We note that the critical point $x=\frac{a}{\sqrt{r^{2}-1}}$ on the short edge corresponding to $S R(a)$ was only used in the situation of Theorem 1.2(b) where $a=b$ and $r=\sqrt{2}$, when it generated the same path as $S$ and $R S$, so this critical point is not needed. That is, swimming from the long side then running 


\section{MINIMIZING TIMES BETWEEN BOUNDARY POINTS ON POOLS}

along the short side will never be optimal, except in the case where $a=b$ and $r=\sqrt{2}$.

Furthermore, by interchanging the roles of $a$ and $b$, we may drop the assumption that $a \geq b$. The results for $r \geq \sqrt{2}$ remain the same, but for $r<\sqrt{2}$, if $b>a$, then interchanging $a$ and $b$ would give a minimal path from running $A=(0, a)$ to $\left(\frac{a}{\sqrt{r^{2}-1}}, 0\right)$ then swim to $B=(b, 0)$ provided $\frac{a}{\sqrt{r^{2}-1}} \leq b$, as seen in the reflections and relabeling of Figure 3. Solving the latter inequality for $a$, we may now summarize the results with no restriction on the relative size of $a$ and $b$.
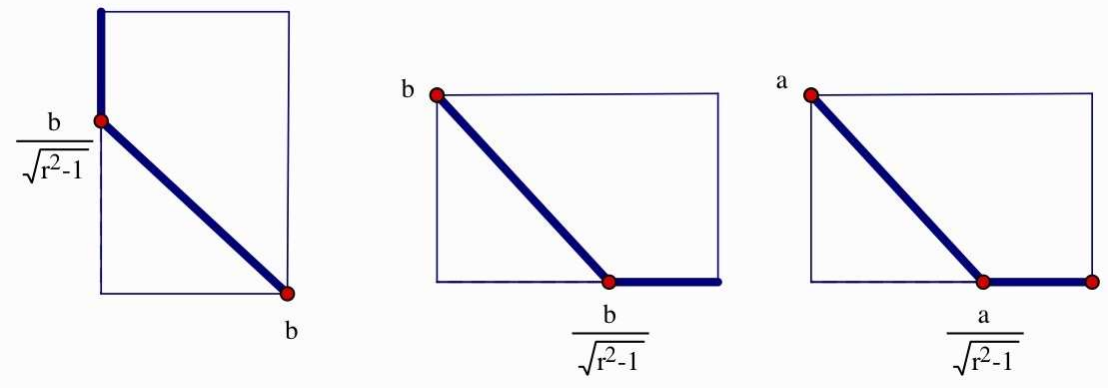

Figure 3. Reflecting over $y=x$ and relabeling for the case $a<b$.

Corollary 1.3. Suppose $A=(0, a)$ and $B=(b, 0)$ with $a, b>0$ are on adjacent edges of a rectangular pool with vertex at $(0,0)$, where the running speed is $r>1$ and the swimming speed is 1 .

(a) For $1<r<\sqrt{2}$, the swim-run path from $A$ to $\left(\frac{a}{\sqrt{r^{2}-1}}, 0\right)$ to $B$ is optimal if $a \leq b \sqrt{r^{2}-1}$, the all-swim path from $A$ to $B$ is optimal if $b \sqrt{r^{2}-1} \leq a \leq \frac{b}{\sqrt{r^{2}-1}}$, and the run-swim path from $A$ to $\left(0, \frac{b}{\sqrt{r^{2}-1}}\right)$ to $B$ is optimal if $a \geq \frac{b}{\sqrt{r^{2}-1}}$.

(b) For $r=\sqrt{2}$, the all run path from $A$ to $(0,0)$ to $B$ is optimal, as are any paths which swim across a $45^{\circ}$ corner.

(c) For $r>\sqrt{2}$, the all run path from $A$ to $(0,0)$ to $B$ is optimal.

\section{The 3-Sided CASE}

Suppose now that we wish to find the fastest path from $A=(0, a)$ on the left side of a rectangular pool $[0, b] \times[0, \infty)$ to a point $C=(b, c)$ on the right side. Again, if the swimming rate $s$ is greater than or equal to the running rate $r$, then the all swimming path is the shortest path, all traced in the fastest medium, and thus would be optimal. Thus, we will again 


\section{T. MIICK AND T. RICHMOND}

assume $r>s=1$. We will classify the paths into to categories: those that contain points on the adjacent "bottom" edge and those that do not.

2.1. Paths avoiding the bottom edge. We will first consider those paths that do not contain points on the bottom edge. If $A$ and $C$ are horizontally aligned, then the optimal path will be the all-swim path, since any other path will involve a longer swim time plus some running time. Assume $A$ and $C$ are not horizontally aligned, so $a \neq c$. Without loss of generality, we will assume $a>c$ and further that $a-c=1$. Thus, with a possible vertical translation, the problem reduces to finding the fastest path from $A=(0,1)$ to $C=(b, 0)$. We note that a run-swim-run path has the same time as a run-swim path or a swim-run path with parallel swim path. Thus we only need to consider the run-swim paths. Running from $A=(0,1)$ to $(0, y)$ then swimming to $C=(b, 0)$ has time function

$$
t(y)=\frac{1-y}{r}+\sqrt{y^{2}+b^{2}} \text { for } y \in[0,1] .
$$

The critical point of $t$ is $y=\frac{b}{\sqrt{r^{2}-1}}$, which falls in the domain $[0,1]$ if and only if $b \leq \sqrt{r^{2}-1}$. Furthermore, $t(y)$ is decreasing on the interval $\left[0, \frac{b}{\sqrt{r^{2}-1}}\right]$ to the left of the critical point and increasing to the right, so $t(0)$, corresponding to the path that crosses the pool perpendicularly, is never minimum, the run-swim path with time $R S(b)=t\left(\frac{b}{\sqrt{r^{2}-1}}\right)=\frac{1}{r}(1+$ $\left.b \sqrt{r^{2}-1}\right)$ is minimum if $b \leq \sqrt{r^{2}-1}$, and the all swim path with time $S(b)=t(1)=\sqrt{1+b^{2}}$ is minimum if $b>\sqrt{r^{2}-1}$.

Lemma 2.1. With a swimming rate of $s=1$ and a running rate of $r>1$, among the paths from $A=(0,1)$ to $C=(b, 0)$ on opposite edges of the rectangle $[0, b] \times[0, \infty)$ which do not touch the bottom edge,

(a) If $r<\sqrt{1+b^{2}}$ (or equivalently, $b>\sqrt{r^{2}-1}$ ), then the all swim path with time $S(b)=t(1)=\sqrt{1+b^{2}}$ is optimal.

(b) If $r \geq \sqrt{1+b^{2}}$ (or equivalently, $b \leq \sqrt{r^{2}-1}$ ), then the run-swim path with time $R S(b)=t\left(\frac{b}{\sqrt{r^{2}-1}}\right)=\frac{1}{r}\left(1+b \sqrt{r^{2}-1}\right)$ is optimal.

For the general case $a>c$, without the assumption that $a-c=1$, we simply scale the lengths above and thus the times by $a-c$ to get the following.

Corollary 2.2. Among the paths which do not contain points on the bottom side, the optimal path from $(0, a-c)$ to $\left(b^{\prime}, 0\right)$ on opposite sides of a rectangle $\left[0, b^{\prime}\right] \times[0, \infty)$ where $b^{\prime}=b(a-c)$, is the path running from $(0, a-c)$ to $\left(0, \frac{b^{\prime}}{\sqrt{r^{2}-1}}\right)$ then swimming to $\left(b^{\prime}, 0\right)$, unless $\frac{b^{\prime}}{\sqrt{r^{2}-1}}>a-c$, in which case the path swimming directly from $(0, a-c)$ to $\left(b^{\prime}, 0\right)$ is optimal. The times 


\section{MINIMIZING TIMES BETWEEN BOUNDARY POINTS ON POOLS}

for such paths are $\frac{a-c}{r}\left(1+b \sqrt{r^{2}-1}\right)$ for the run-swim path if $b \leq \sqrt{r^{2}-1}$, and $(a-c) \sqrt{1+b^{2}}$ for the all-swim path if $b \geq \sqrt{r^{2}-1}$.

Similar scaling is possible on our further results, which we will not state in such full generality. At the boundaries of piecewise criteria, the optimal path may arise from two of the functions in Lemma 1.1. For simplicity, we do not always mention such duplication.

2.2. Paths touching the bottom edge. Now let us consider the paths from $A=(0, a)$ to $C=(b, c)$ on opposite sides of the rectangle $[0, b] \times[0, \infty)$ which include a point $B=(x, 0)$ on the bottom edge. The optimal such path must be optimal from $A$ to $B$ and from $B$ to $C$, and each of these is an optimal path between points on adjacent sides, as considered in the previous section.

If $r \geq \sqrt{2}$, then the all running paths from $A$ to $B$ and from $B$ to $C$ are optimal, so the all running path will be optimal from $A$ to $C$. And furthermore, in the case $r=\sqrt{2}$, the minimum time of $\frac{a+b+c}{r}$ is also achieved by paths which cut off $45^{\circ}$ corners.

Now suppose $1<r<\sqrt{2}$. We have two cases based on the distance $b$ between the opposite sides.

Case 1: $b>\frac{a+c}{\sqrt{r^{2}-1}}$, or equivalently, $r>\sqrt{1+\left(\frac{a+c}{b}\right)^{2}}$. In this case, the critical point for the swim-run path from $A$ to the bottom occurs to the left of the critical point for the swim-run path from $C$ to the bottom, allowing these paths to connect for an optimal swim-run-swim path. Formally, either (a) the point $B$ is farther than $\frac{a}{\sqrt{r^{2}-1}}$ from the left endpoint $(0,0)$ of the bottom edge, or (b) $B$ is farther than $\frac{c}{\sqrt{r^{2}-1}}$ from the right endpoint $(b, 0)$ of the bottom edge. Suppose (a). Now the fastest path from $A$ to $C$ through $B$ necessarily starts with the fastest path from $A$ to $B$, and by the two-sided problem, we know that this path swims from $A$ to $B^{\prime}=\left(\frac{a}{\sqrt{r^{2}-1}}, 0\right)$ then runs to $B$. Now this path passes through $B^{\prime}$, which is more than $\frac{c}{\sqrt{r^{2}-1}}$ from the right endpoint $(b, 0)$ of the bottom edge, and ends at $C$. For this path to be optimal from $B^{\prime}$ to $C$, by the two-sided analysis it must run from $B^{\prime}$ to the point $B^{\prime \prime}=\left(b-\frac{c}{\sqrt{r^{2}-1}}, 0\right)$ which is $\frac{c}{\sqrt{r^{2}-1}}$ units from $(b, 0)$, then swim to $C=(c, b)$. The case (b) is similar. Thus, the optimal path is a swim-run-swim path from $A$ to $B^{\prime}$ to $B^{\prime \prime}$ to $C$. The time for this path is $S R S(b)=\frac{1}{r}\left(b+(a+c) \sqrt{r^{2}-1}\right)$.

Case 2: $b \leq \frac{a+c}{\sqrt{r^{2}-1}}$, or equivalently, $r \leq \sqrt{1+\left(\frac{a+c}{b}\right)^{2}}$. In this case, the critical point for the swim-run path from $A$ to the bottom is too far to the right to allow an optimal swim-run path from $C$ to the bottom to connect with the optimal swim-run path from $A$. We will see that in this case, no path containing a point on the bottom edge is optimal. Formally, we have 


\section{T. MIICK AND T. RICHMOND}

$b-\frac{c}{\sqrt{r^{2}-1}}<\frac{a}{\sqrt{r^{2}-1}}$. Now either (a) the included point $B$ on the bottom edge is no more than $\frac{a}{\sqrt{r^{2}-1}}$ to the left edge, (b) the included point bottom edge point $B$ is no more than $\frac{c}{\sqrt{r^{2}-1}}$ to the right edge, or (c) both.

Suppose only (a) holds. Then applying the two-sided analysis, the optimal path from $A$ to $B$ is either an all-swim path or a swim-run path arriving at $B$ by swimming from a point $A^{\prime}$ on the left edge. Since (b) does not hold, the minimal path from $C$ to $B$ is a run-swim path from $C$ to $B^{\prime}=\left(b-\frac{c}{\sqrt{r^{2}-1}}, 0\right)$ to $B$. But if this path from $A$ to $B$ to $B^{\prime}$ to $C$ is minimal, the path from $A$ to $B^{\prime}$ must be minimal, and since $b-\frac{c}{\sqrt{r^{2}-1}}<\frac{a}{\sqrt{r^{2}-1}}$, the minimal path from $A$ to $B^{\prime}$ arrives at $B^{\prime}$ by swimming. Thus, $B=B^{\prime}$. Now the minimal path involves swimming from a point $A^{\prime}$ on the left edge to $B=B^{\prime}$ then swimming on to $C$. The swimming portion of this path can be done faster by swimming directly from $A^{\prime}$ to $C$, so this is not optimal.

If only (b) holds, the symmetric argument obtained by interchanging $a$ and $c$ above shows that running to a point $B$ on the bottom edge is not minimal.

If both (a) and (b) hold, then the minimal paths from $A$ to $B$ and from $C$ to $B$ both arrive at $B$ by swimming, either in an all-swim path or a runswim path from points $A^{\prime}$ and $C^{\prime}$ on the left and right edges, respectively. Again, swimming directly from $A^{\prime}$ to $C^{\prime}$ without detouring through the point $B$ is faster, so this is not optimal. Thus, in Case 2, an optimal path will never hit the bottom edge.

We summarize our results.

Lemma 2.3. With a running rate of $r>1$ and swimming rate $s=1$, the minimal time for a path from $A=(a, 0)$ on one side of a rectangular pool $[0, b] \times[0, \infty)$ to a point $B$ on the bottom and on to $C=(b, c)$ on the opposite side is determined as follows:

(a) if $\sqrt{1+\left(\frac{a+c}{b}\right)^{2}}<r<\sqrt{2}$, the swim-run-swim path with time $S R S(b)=$ $\frac{1}{r}\left(b+(a+c) \sqrt{r^{2}-1}\right)$ is minimum.

(b) If $r=\sqrt{2}$, the all running path or any path that swims across one or two $45^{\circ}$ corners is minimum, with time $\frac{a+b+c}{r}$.

(c) If $r>\sqrt{2}$, the all running path with time $\frac{a+b+c}{r}$ is minimum.

Furthermore, if $1<r \leq \sqrt{1+\left(\frac{a+c}{b}\right)^{2}}$, then the optimal path from $A$ to $C$ will not include any point on the bottom.

2.3. Comparing paths touching or avoiding the bottom edge. Having optimized paths from $A=(0, a)$ to $C=(b, c)$ which do or do not contain points $B=(x, 0)$ on the bottom edge, we will now compare all such paths to find the optimal path from $A$ to $C$. 


\section{MINIMIZING TIMES BETWEEN BOUNDARY POINTS ON POOLS}

We first address the case $a=c$ of horizontally aligned points.

Theorem 2.4. Suppose $A=(0, a)$ and $C=(b, a)$ are horizontally aligned points on opposite sides of a rectangular pool $[0, b] \times[0, \infty)$.

(a) If $r \geq \sqrt{2}$, the all swimming path with time $b$ is optimal if and only if $b \leq \frac{2 a}{r-1}$ if and only if $r \leq 1+\frac{2 a}{b}$. Otherwise, the all running with time $\frac{2 a+b}{r}$ is optimal.

(b) If $1<r<\sqrt{2}$, the swim-run-swim path with time $S R S(b)=\frac{1}{r}(b+$ $2 a \sqrt{r^{2}-1}$ ) is optimal if and only if $b \geq \frac{2 a \sqrt{r^{2}-1}}{r-1}=2 a \sqrt{1+\frac{2}{r-1}}$. Otherwise, the all swimming path with time $b$ is optimal. In particular, all swimming is optimal if $b<2 a$.

Proof. With $A$ and $C$ vertically aligned, the fastest path not hitting the bottom was the path swimming directly, $b$ units, requiring $b$ units of time. For $r \geq \sqrt{2}$, the fastest path hitting the bottom edge is all running, which takes $\frac{2 a+b}{r}$ units of time, so the all-swim path is optimal if and only if $b \leq \frac{2 a+b}{r}$ if and only if $b \leq \frac{2 a}{r-1}$ if and only if $r \leq 1+\frac{2 a}{b}$. This proves (a).

For $r<\sqrt{2}$, we must compare the swim-run-swim time $\frac{1}{r}\left(b+2 a \sqrt{r^{2}-1}\right)$ with the all swim time $b$. Now $\frac{1}{r}\left(b+2 a \sqrt{r^{2}-1}\right)>b$ if and only if $b \geq$ $\frac{2 a \sqrt{r^{2}-1}}{r-1}$. However, the swim-run-swim path is only valid if $b>\frac{2 a}{\sqrt{r^{2}-1}}$. But since $r>1$, we have $r-1<r^{2}-1$, so $\frac{2 a \sqrt{r^{2}-1}}{r-1}>\frac{2 a \sqrt{r^{2}-1}}{r^{2}-1}=\frac{2 a}{\sqrt{r^{2}-1}}$. So, $b \geq \frac{2 a \sqrt{r^{2}-1}}{r-1}$ implies $b>\frac{2 a}{\sqrt{r^{2}-1}}$, so that the swim-run-swim is indeed valid. This completes the proof of (b).

Now we consider non-horizontally aligned points $A$ and $C$. Again we will assume $a>c$ and scale the problem so that $a-c=1$. We will present separate theorems for the cases $1<r<\sqrt{2}$ and $r \geq \sqrt{2}$.

Theorem 2.5. If $1<r<\sqrt{2}$ and $a-c=1$, the minimal time for a path from $A=(a, 0)$ to $C=(b, c)$ on opposite sides of a rectangular pool $[0, b] \times[0, \infty)$ is determined as follows:

(a) If $1<r<\sqrt{1+b^{2}}$ and $b \leq \frac{(2 c+1)+2 r \sqrt{c(1+c)}}{\sqrt{r^{2}-1}}$, then the all swim path with time $S(b)=\sqrt{1+b^{2}}$ is minimum.

(b) If $1<r<\sqrt{1+b^{2}}$ and $b \geq \frac{(2 c+1)+2 r \sqrt{c(1+c)}}{\sqrt{r^{2}-1}}$, then the swim-run-swim path with time $S R S(b)=\frac{1}{r}\left(b+(2 c+1) \sqrt{r^{2}-1}\right)$ is minimum.

(c) If $r \geq \sqrt{1+b^{2}}$, then the run-swim path with time $R S(b)=\frac{1}{r}(1+$ $\left.b \sqrt{r^{2}-1}\right)$ is minimum.

Proof. Suppose $1<r<\sqrt{2}$. If $r<\sqrt{1+b^{2}}$, then $S(b)$ is the optimal time not hitting the bottom and if $b>\frac{2 c+1}{\sqrt{r^{2}-1}}$, the path to the bottom 


\section{T. MIICK AND T. RICHMOND}

with time $S R S(b)$ may be optimal. The line $S R S(b)$ intersects the concave up hyperbola $S(b)$ at $b=\frac{(2 c+1) \pm 2 r \sqrt{c(1+c)}}{\sqrt{r^{2}-1}}$, so $S(b)$ is below $S R S(b)$ only between these two intersection points. But, $S R S(b)$ is not valid to the left of $\frac{(2 c+1)-2 r \sqrt{c(1+c)}}{\sqrt{r^{2}-1}}<\frac{2 c+1}{\sqrt{r^{2}-1}}$, so $S R S(b)$ is optimal only for $b \geq \frac{(2 c+1)+2 r \sqrt{c(1+c)}}{\sqrt{r^{2}-1}}$.

For $r \geq \sqrt{1+b^{2}}$, we only need to compare the optimal time $R S(b)$ not hitting the bottom and, if $b>\frac{2 c+1}{\sqrt{r^{2}-1}}$, the optimal time $S R S(b)$ hitting the bottom. Now $R S(b)$ and $S R S(b)$ are linear functions of $b$ which intersect at $b=P=\frac{1-(2 c+1) \sqrt{r^{2}-1}}{1-\sqrt{r^{2}-1}}$. Since $R S(b)$ has the smaller slope, $R S(b)<$ $S R S(b)$ for all points $b$ to the right of the intersection point $P$. Letting $x=\sqrt{r^{2}-1}$, and noting $c>0$ and $0<\sqrt{r^{2}-1}<1$, we see that if $\operatorname{SRS}(b)$ is an option, then $b>\frac{2 c+1}{\sqrt{r^{2}-1}}>\frac{1}{\sqrt{r^{2}-1}}>1>\frac{1-(2 c+1) x}{1-x}=P$, so $b$ is to the right of $P$ and $R S(b)<S R S(b)$.

Now we consider the case for $r \geq \sqrt{2}$.

Theorem 2.6. If $r \geq \sqrt{2}$ and $a-c=1$, the minimal time for a path from $A=(a, 0)$ to $C=(b, c)$ on opposite sides of a rectangular pool $[0, b] \times[0, \infty)$ is determined as follows:

(a) If $\sqrt{2} \leq r<\sqrt{1+b^{2}}$, and $b \leq 2 c+1=a+c$, the all swim path with time $S(b)=\sqrt{1+b^{2}}$ is minimum for $b$ between $\frac{(2 c+1) \pm r \sqrt{(2 c+1)^{2}+1-r^{2}}}{r^{2}-1}$, and the all run path (possibly with swimming across $45^{\circ}$ corners if $r=\sqrt{2}$ ) with time $R(b)=\frac{2 c+1+b}{r}$ is minimum otherwise.

(b) If $\sqrt{2} \leq r<\sqrt{1+b^{2}}$, and $b>2 c+1=a+c$, then the all run path (possibly with swimming across $45^{\circ}$ corners if $r=\sqrt{2}$ ) with time $R(b)=\frac{2 c+1+b}{r}$ is minimum.

(c) If $r>\sqrt{2}$ and $r \geq \sqrt{1+b^{2}}$, then the all run path with time $R(b)=$ $\frac{2 c+1+b}{r}$ is minimum if $b>\frac{2 c}{\sqrt{r^{2}-1}-1}$, and the run-swim path with time $R S(b)=\frac{1}{r}\left(1+b \sqrt{r^{2}-1}\right)$ is minimum otherwise.

(d) If $r=\sqrt{2}$ and $b \leq 1$ so that $r \geq \sqrt{1+b^{2}}$, then the run-swim path with time $R S(b)=\frac{1}{r}\left(1+b \sqrt{r^{2}-1}\right)$ is minimum.

Proof. Suppose $r \geq \sqrt{2}$.

If $\sqrt{2} \leq r<\sqrt{1+b^{2}}$, then we need to compare the optimal times $R(b)$ and $S(b)$ for paths hitting and not hitting the bottom, respectively. Equat-

ing $R$ and $S$, we find that they intersect when $b=\frac{2 c+1 \pm r \sqrt{(2 c+1)^{2}+1-r^{2}}}{r^{2}-1}$, provided the discriminant is nonnegative. The curves do not intersect if and only if the discriminant $(2 c+1)^{2}+1-r^{2}$ is negative, which occurs precisely when $r>\sqrt{(2 c+1)^{2}+1}$, which implies $r>2 c+1$. In this case, 


\section{MINIMIZING TIMES BETWEEN BOUNDARY POINTS ON POOLS}

$R(0)=\frac{2 c+1}{r}<1=S(0)$, so $R(b)$ is always below $S(b)$ if they do not intersect. If the curves do intersect, since $S$ is concave up, $S(b)$ is below $R(b)$ only between these two intersection points. The curves do intersect if and only if $r<\sqrt{(2 c+1)^{2}+1}$. Thus, the requirement that $r<\sqrt{b^{2}+1}$ implies the curves do not intersect if $2 c+1<b$. This proves (b). If $2 c+1 \geq b$, then the curves intersect and we have the result of (a).

If $r \geq \sqrt{1+b^{2}}$, the optimal paths hitting and not hitting the bottom are $R(b)$ and $R S(b)$. Notice that $R(b)$ and $R S(b)$ are linear functions. If $r>\sqrt{2}, R S(b)$ has the larger slope, so $R S(b)$ will be below $R(b)$ to the left of their intersection point $b=\frac{2 c}{\sqrt{r^{2}-1}-1}$, proving (c). If $r=\sqrt{2}$ then $R S(b)<R S(b)+\frac{2 c}{\sqrt{2}}=R(b)$, so $R S(b)$ is minimum, proving (d).

\section{THE 4-SIDED CASE}

If we have points $A=(0, a)$ and $C=(b, c)$ on opposite sides of a rectangle $[0, b] \times[0, d]$, we have considered the case of getting from $A$ to $C$ optimally with the possibility of hitting the bottom edge. Hitting the bottom edge was never optimal if $b \leq \frac{a+c}{\sqrt{r^{2}-1}}$ where $a$ and $c$ were the distances along the vertical edges from $A$ and $C$ respectively to the bottom. If $a^{\prime}$ and $c^{\prime}$ are the vertical distances from $A$ and $C$ to the top, the paths that hit the top reduce to the previously considered 3 -sided case (rotated $180^{\circ}$ ). Let us assume $a+c<a^{\prime}+c^{\prime}$. Then we should consider where $b$ falls among the points $\frac{a+c}{\sqrt{r^{2}-1}}<\frac{a^{\prime}+c^{\prime}}{\sqrt{r^{2}-1}}$. If $b$ is smaller than both, then neither paths to the top nor the bottom are optimal. If $b$ is only smaller than $\frac{a^{\prime}+c^{\prime}}{\sqrt{r^{2}-1}}$, then only the paths to the bottom may be optimal. If $b$ is larger than both, then the minimal path to the bottom takes $\frac{1}{r}\left(b+(a+c) \sqrt{r^{2}-1}\right)$ units of time, which is less than the minimal time $\frac{1}{r}\left(b+\left(a^{\prime}+c^{\prime}\right) \sqrt{r^{2}-1}\right)$ for a path hitting the top. Thus, in the 4 -sided case, we only need to consider paths reaching the horizontal side - top or bottom - which is "closest" in the sense that the sum of the vertical distances from that side to $A$ and to $C$ is smallest, and apply the 3 -sided analysis.

\section{REFERENCES}

[1] K. Bell, S. Polson, and T. Richmond, The fastest path between two points, with a symmetric obstacle, The College Math. J., 46.2 (2015), 92-97.

[2] M. Golomb, Elementary proofs for the equivalence of Fermat's principle and Snell's law, The American Mathematical Monthly, 71.5 (1964), 541-543.

[3] P. Mandrekar and T. Josepy, When to cut corners and when not to, The Mathematical Intelligencer, 36 (2014), 70-74.

[4] T. Miick, Minimizing Travel Time Through Multiple Media with Various Borders, Masters Thesis, Western Kentucky University, May 2013: http://digitalcommons.wku.edu/cgi/viewcontent. cgi ?article $=2249 \&$ context $=$ theses . 


\section{T. MIICK AND T. RICHMOND}

[5] T. J. Pennington, Do dogs know calculus?, The College Math. J., 34.3 (2003), 178-182; http://www.maa.org/features/elvisdog.pdf.

[6] D. Pedoe, A geometric proof of the equivalence of Fermat's principle and Snell's law, The American Mathematical Monthly, 71.5 (1964), 543-544.

MSC2010: 26A06, 65K10

Key word: optimization

Department of Mathematics, Western Kentucky University, 1906 College Heights Blvd., Bowling Green, KY 42101

E-mail address: tonja.miick@wku.edu

Department of Mathematics, Western Kentucky University, 1906 College

Heights Blvd., Bowling Green, KY 42101

E-mail address: tom.richmond@wku.edu 